\title{
Pasteurella multocida meningitis in an adult: case report
}

\author{
G R Armstrong, R A Sen, J Wilkinson
}

\begin{abstract}
Pasteurella multocida is known to form part of the normal flora in the nasopharynx or gastrointestinal tract in many domestic and wild animals. Most human $P$ multocida infections are soft tissue infections caused by dog or cat bites. Less commonly this bacterium is associated with infections affecting other organ systems of man. A case of fatal $P$ multocida meningitis discovered at the necropsy of a 52 year old man is described. $P$ multocida is an unusual causative agent of meningitis which tends to affect those at the extremes of age.

(F Clin Pathol 2000;53:234-235)
\end{abstract}

Keywords: Pasteurella multocida; meningitis

Pasteurella multocida is a non-spore forming, non-motile, Gram negative coccobacillus. It has been isolated from the respiratory tract and digestive system of domestic pets, livestock, and many wild animals. The carriage rate of the organism is believed to be up to $90 \%$ in cats and up to $66 \%$ in dogs. ${ }^{1} P$ multocida has been known to cause infections in humans, the most familiar being soft tissue infection following animal bites. However, this organism may affect a variety of systems causing serious disease.

\section{Case report}

A 52 year old man was found dead at home. No history of illness in the preceding days was available, there is no record of him seeking any medical attention. He had recently been under investigation for dyspepsia but otherwise had no medical history of serious illness. The subject lived with his brother, both reportedly in a state of serious self neglect. Their house was soiled with dog faeces throughout and their pet dog was in poor health.

Department of Histopathology, Hope Hospital, Stott Lane, Salford, Manchester M6 8HD, UK J Wilkinson

Department of Microbiology, Hope Hospital

G R Armstrong

R A Sen

Correspondence to: Dr Wilkinson

Accepted for publication 11 August 1999

\section{Pathological examination}

The subject was a white male whose appearance was consistent with the stated age of 52 years. He was well nourished and there were no external marks, bites, or scratches. On internal examination a purulent meningitis was found. In particular the cranial vertex and base of brain were affected. A sample of meningeal pus was taken for subsequent culture. The brain was slightly swollen and there was generalised vascular congestion. No skull fractures were present.

The lungs were congested and mildly oedematous. A small thromboembolus was present in the right lower lobe pulmonary artery. There was no evidence of chronic respi- ratory disease. Mild coronary atherosclerosis and duodenitis were noted. There was no evidence of malignancy, and no other foci of infection were identified. The cause of death was declared to be purulent meningitis.

\section{Bacteriology}

Postmortem culture of a swab of meningeal pus on chocolate blood agar produced a pure growth of colonies resembling Haemophilus spp within 24 hours. However, the isolate was identified on API 20 NE (Bio-Merieux Ltd) as Pasteurella multocida.

\section{Discussion}

Pasteurellae were first observed in the blood of birds with fowl cholera in 1877. P multocida ("killer of many species") was isolated by Pasteur as the causative agent of this disease in 1880. Human infection by $P$ multocida was not recognised until some years later. The first case reported was described in 1913 by Brugnatelli. ${ }^{1}$ In this case the organism was isolated from the blood of a woman with puerperal fever.

$P$ multocida is most commonly recognised as a cause of soft tissue infection secondary to animal bites. However, various infections have been described where this organism was the agent responsible, in particular affecting those with impaired immune defences and those at the extremes of age. ${ }^{12}$

$P$ multocida meningitis is uncommon. Cases of $P$ multocida meningitis have occurred following animal bites, scratches, and other animal contact. The organism has also been known to be transmitted through animal saliva without a bite having occurred. ${ }^{3}$ In some cases of infection, no known animal contact has been determined. ${ }^{14}$ Meningitis caused by this agent has also been reported following spread of the bacterium from infected wounds after craniofacial surgery. ${ }^{1} \mathrm{~A}$ few cases have occurred in neonates after infection related to maternal carriage of the organism in the genital tract. ${ }^{5}$

The subjects in whom $P$ multocida meningitis most often occurs are at the extremes of age, being either elderly people or young infants. The typical case involves infants under the age of one year with a history of animal exposure, and cases in adults are unusual. ${ }^{1-5}$ Kumar et al, in a review of cases, found that $40 \%$ occurred in infants under one year. When post-traumatic and post-surgical cases were eliminated, $70 \%$ of adult cases occurred in people over 55 years of age. ${ }^{2}$

Mortality in P multocida meningitis is high. In the series by Kumar overall mortality was $30 \%$, but was higher in the elderly- $63 \%$ in patients over 60 years in age. ${ }^{2}$ In another series 
of 17 cases, seven of whom were adults, six patients died within 72 hours of hospital admission. Four of these six fatalities involved persons over 60 years of age.

Diagnosis of this agent can be difficult, in that Gram staining of CSF will reveal a Gram negative coccobacillus. This results in the organism being confused with more common agents of meningitis such as $N$ meningitidis and $H$ influenzae. However, identification of $P$ multocida is normally straightforward, although false positive antigen testing with latex particle agglutination tests has been reported. ${ }^{3}$

In conclusion, we have presented a case of meningitis caused by $P$ multocida, causing a sudden death in the community. The subject was an adult with no evidence of recent animal bite or chronic debility, but who lived in squalor with obvious animal contamination of his environment. Cases such as this appear to be unusual. However, the high frequency of animal contacts and the relative rarity of such infections must indicate that the risk is low. The majority of infections are wound infections related to animal bites. P multocida meningitis is rare, particularly in the adult. However, it is a very serious condition with a high mortality. Therefore, where meningitis occurs in addition to a history of animal exposure, $P$ multocida infection should be considered.

Acknowledgements to HM Coroner, Bolton, for the kind permission to report details of the above case.

1 Weber DJ, Wolfson JS, Swartz MN, et al. Pasteurella multocida infections. Report of 34 cases and review of the literature. Medicine (Baltimore) 1984;63:133-54.

2 Kumar A, Devlin R, Velland H. Pasteurella meningitis in an adult: case report and review. Rev Infect Dis 1990;12:440-8.

3 Costa-Cruz O, Sesso AM, Mate S, et al. Pasteurella multocida meningitis. $N$ fersey Med 1990;87:127-9.

4 Boocock GR, Bowley JA. Meningitis in infancy by Boocock GR, Bowley JA. Meningitis in
Pasteurella multocida. F Infect 1995;31:161-2.

5 Hillery S, Reiss-Levy E, Browne C, et al. Pasteurella multocida meningitis in a two day old neonate. Scand $\mathcal{F}$ Infect Dis 1993;25:655-8. 


\section{Letters}

133, Milan, Italy

P A NUTTALL

$\mathrm{R} J$ SOKOL

NBS Trent Centre, Sheffield S5 7fN, UK

Prevalence of HIV and hepatitis C markers among a cadaveric population in Milan

Cattaneo et al report the interesting finding of human immunodeficiency virus (HIV) infection in individuals lacking risk behaviour. With any postmortem sample, serological false positivity is a well known phenomenon,,$^{2-3}$ and in the above instance in particular, confirmatory testing is important for confidence in test results. Although concordance in different screening assays is an excellent predictor for true positivity with any antemortem blood samples, the same may not be true for postmortem samples, where false positivity as a result of unsuitability of analyte can occur with more than one assay. When a single screening test is used, confirmation is especially important.

JOHN BARBARA

London and SE Zone NBS, London, UK

GEORGE GALEA

Scottish National Blood Transfusion Service, 21 Ellen's Glen Road, Edinburgh EH17 7QT, UK RUTH WARWICK

London and SE Zone NBS, London, UK

1 Cattaneo C, Nuttall PA, Molendini LO, et al. The prevalence of HIV and hepatitis $\mathrm{C}$ markers among a cadaveric population of Milan. 7 Clin Pathol 1999;52:267-70.

2 Heim A, Wagner D, Rothamel $\mathrm{T}$, et al. Evaluation of serological screening of cadaveric sera for donor selection for cornea transplantation. 7 Med Virol 1999;58:291-5.Please provide tion. $7 \mathrm{Med}$ irol $1999 ; 58$ :

3 Burtonboy G, Delloye C. Polymerase chain reaction in cadaveric blood and tissue. Transplant Proc 1996;28:2927-8.

\section{The authors reply}

We thank Drs Barbara, Galea, and Warwick for their letter concerning our article on testing specimens from cadavers for markers of human immunodeficiency virus (HIV) and hepatitis $\mathrm{C}$ infection. The points they raise are all covered in the article, but are worth emphasising.

We are well aware that false positive serological reactions can occur with postmortem samples and commented on this in the discussion section; it was for this reason that data for hepatitis B serology were not included.

We agree that confirmation testing is especially important and were pleased with the concordance of the postmortem results for HIV testing between the enzyme linked immunosorbent assay (ELISA) and agglutination methods, and between the postmortem and (where known) antemortem findings.

Our article emphasises that in the subjects studied, lack of risk behaviour does not mean that there was necessarily no risk behaviourjust that it was not common knowledge and was unknown to the pathologist carrying out the necropsy.

The main point of our paper was that medico-legal practice deals with a particular and selected population that has a high prevalence of markers for HIV and hepatitis $\mathrm{C}$ infection, irrespective of known risk behaviour, and in many cases a rapid and easily performed screening test would give early warning of a potential problem.

\section{Notices}

\section{Update in Surgical Pathology II} 12-16 fuly 2000

Insitute of Pathology, Wurzburg, Germany

This course will be held jointly by the Department of Pathology, Massachusetts General Hospital and Harvard Medical School, Boston, Massachusetts, USA and the Department of Pathology, University of Wurzburg, Germany. The course will Topics will include pathology of the lung, liver, thymus, thyroid, breast, ovary, endometrium, skin, gastrointestinal tract, head and neck, kidney, bladder, lymph nodes, heart, and immunohistochemistry.

Further information from: Dr Eugene J Mark, Department of Pathology, 55 Fruit Street, Massachusetts General Hospital, Boston, Massachusetts 02114, USA; fax: +1 6177267474 ; email: emelchionno@partners.org (for North and South America). Professor HK Muller-Hermelink, Department of Pathology, University of Wurzburg, Luitpoidkrankenhaus, Josef-SchneiderStrabe 2, Germany; fax: +49 931201 3440; email: path161@mail. uniwuerzburg. de (for Europe, Asia, Africa, and Australia)

\section{9th Dermatopathology Self Assessment Workshop \\ 15-16 September 2000}

Catholic University Medical School

(Instituti Biologici), Rome, Italy

Forty cases of connective tissue neoplasms and 40 cases of melanocytic skin neoplasms will be provided and illustrated by Professor CDM Fletcher (Harvard University, Boston, USA) and Professor RL Barnhill (Pathology Services, Boston, USA), respectively. During the meeting and the days before, histological slides of all cases will be available for hands on educational sessions at the microscope. There will also be short updates regarding a few newly described mesenchymal and melanocytic neoplasms of the skin. (Official language, English.)

Fee for the workshop $\$ 200$.

Further information from: Professor Guido Massi, Department of Pathology, Catholic University Medical School, Largo F. Vito, 1-00168 Rome, Italy; tel: +3906 305 2345, 350 3481; fax: +3906 305 1343; email: guidomassi@tiscalinet.it include lectures as well as workshops.

\section{Correction}

Pasteurella multocida meningitis in an adult: case report. Armstrong GR, Sen RA, Wilkinson J. $\mathcal{f}$ Clin Pathol 2000;53:234-5.

\section{Book reviews}

Cerebral Ischemia: Molecular and Cellular Pathophysiology. Walz W, ed. (\$125.00.) Humana Press, 1999. ISBN 0 896035409.

This book reviews the mechanisms of neuronal damage and the accompanying cellular reactions that are triggered by cerebral ischaemia. The contributors are largely based in North America, and many have a distinguished record in this field. The first contribution is a very useful overview of the mechanisms of cerebral ischaemic damage, which is followed by five sections that focus on neuronal damage, with particularly interesting sections on calcium overload and neuroprotection as potentially mediated by cytokines. The remaining four contributions focus on the cellular changes following ischaemia, the highlights of which for me were an illuminating discussion on necrosis versus apoptosis in neurones, and the reprogramming of gene expression in neurones after ischaemia. The text throughout the book is accompanied by black and white line diagrams and tables, with only occasional monochrome illustrations. The book is well referenced (up to 1998) and there is a helpful index.

Cerebral ischaemia is a major cause of morbidity and mortality in Western countries and so this book will be welcomed by both clinical and basic neuroscientists, neuropathologists, and neurophysiologists. It is rather specialised for most general departmental collections, but I would recommend this as a library purchase. Over the past few weeks the book has been used by undergraduate and postgraduate students, postdocs, and neuropathologists and we have all found it helpful (without being overwhelming) and clearly presented (without being simplistic).

JAMES W IRONSIDE

Clinical Investigation and Statistics in Laboratory Medicine. Jones R, Payne B. (£21.00.) ACB Venture Publications, 1997. ISBN 0902429213.

Statistics is one of those subjects that divides people into two camps. There are those who revel in the abstract beauty of mathematics, sometimes ignoring the inconvenient habit of data to fail to conform to mathematical models. There are others who 
regard statistics as a form of codified guesswork and, at worst, a tool to increase the chance of publication of data beyond a $\mathrm{p}$ value of 0.05 . It is a challenge to write for such a partitioned readership and Jones and Payne have, quite rightly, tended to serve the needs of the less numerate majority.

This book is well laid out with the use of highlighted sections and abundant graphical illustrations. There are few formulae with which to grapple and the text is mainly a narrative account of the statistical manipulations and the reasons behind them. This is leavened with a sprinkling of biographical snippets.

Overall, this is not a "how to do it" text but rather it explains why things are done that way. The volume is relatively slim, there are less than 190 pages of figures and text and this has led to a rather steep rate of ascent in the introductory section. However, for a readership that has a medical background and has endured formal attempts by statisticians to justify their stewardship of the Holy Grail, this text is to be recommended. Those undertaking research projects that require detailed statistical interpretation may then move on to more applied texts such as Altman's Practical Statistics for Medical Research (Chapman and Hall, London 1991, ISBN 041227630 5). However, many may not make this progression and an acquaintance with the contents of Clinical Investigation and Statistics in Laboratory Medicine would, nevertheless, be a major advantage.

WILLIAM R ROCHE

\section{aGp \\ Best Practice articles (formerly "Broadsheets") prepared by the Association of Clinical Pathologists}

Just published

158 Neuropathology $2000 \quad$ WR TIMPERLEY

157 Guidelines for the laboratory handling of laryngectomy specimens 2000 TR HELLIWELL

156 Handling oesophageal biopsies and resection specimens and their reporting 2000 NBN IBRAHIM

\section{Recent Publications}

155 Pathological investigation of deaths following surgery, anaesthesia, and medical procedures 1999 RD START, SS CROSS

154 Helicobacter pylori 1999 CAM MCNULTY, JI WYATT (with correction in June issue)

\section{Other Best Practice articles are still available for purchase}

153 The laboratory investigation of vaginal discharge 1998 KF MACSWEEN, GL RIDGWAY

152 Clinical implications of plasma homocysteine measurement in cardiovascular disease 1998 RA STILL, IFW MCDOWELL

151 Investigation of dyslipidaemias 1997 AF WINDER, W RICHMOND, DT VALLANCE

150 Antenatal serological testing and prevention of haemolytic disease of the newborn 1997 JKM DUGUID

149 Serological diagnosis of gluten sensitive enteropathy 1996 DJ UNSWORTH

148 Laboratory diagnosis of malaria 1996 DC WARHURST, JE WILLIAMS

147 Mycological techniques 1996 KG DAVEY, CK CAMPBELL, DW WARNOCK

146 Macroscopic examination of prostatic specimens 1995 P HARNDEN, MC PARKINSON

145 Investigation of patients with autoimmune haemolytic anaemia and provision of blood for transfusion 1995 RJ SOKOL, DJ BOOKER, R STAMPS
Earlier Broadsheets may still be available from the author. A full list can be obtained from the Publications Secretary, Association of Clinical Pathologists, 189 Dyke Road, Hove, East Sussex BN3 1TL.

\section{Prices}

INLAND One copy, $£ 2.50$; $2-10$ copies (of any one broadsheet or reprint), $£ 2.00$ each; $11-100$ copies (of any one), $£ 1.75$ each; 101 plus copies (of any one), price to be agreed; authors (over 50 free copies), 1.25 each. OVERSEAS One copy, $\$ 6.75 ; 2-10$ copies (of any one broadsheet or reprint), $\$ 5.25 ; 11-100$ copies (of any one), $\$ 3.75 ; 101$ plus copies (of any one), price to be agreed.

Authors $\$ 2.25$. Prices include postage but air mail will be charged extra. Trade discount $10 \%$. All orders (and all changes of address of regular subscribers) should be sent to the Publishing Manager • Fournal of Clinical Pathology, BMJ Publishing Group, BMA House, Tavistock Square, London WC1H 9JR. 